\title{
LÉXICO DISPONIBLE EN TRES CENTROS DE INTERÉS DE APRENDICES DE ESPAÑOL COMO LENGUA EXTRANJERA
}

\author{
AVAILABLE LEXICON IN THREE INTEREST CENTERS AMONG SFL LEARNERS \\ LEXIQUE DISPONIBLE DANS TROIS CENTRES D'INTÉRÊT D'APPRENANTS ELE
}

Lorena Paulina Blanco San Martín

Magíster en Lingüística Aplicada, Universidad de Concepción, Chile. Profesora de Lenguaje y Comunicación, Universidad Católica de la Santísima Concepción. Profesora del "Programa de Español como Lengua Extranjera", Universidad de Concepción, Chile. Facultad de Humanidades y Arte, Departamento de Español, Edificio de Lenguas, Casilla 160-C.

Correo 3, Concepción. Chile. Iblanco@udec.cl

\section{Anita Alejandra Ferreira Cabrera Ph.D. en Computer Science en Artificial Intelligence, Universidad de Edimburgo. Doctora en Lingüística, Universidad Católica de Valparaíso. Profesora titular del Departamento de Español de la Facultad de Humanidades y Artes, Universidad de Concepción, Chile. \\ Facultad de Humanidades y Arte, Departamento de Español, Edificio de Lenguas, Casilla 160-C. \\ Correo 3, Concepción. Chile. aferreir@udec.cl}

Este estudio se desarrolló en el contexto del proyecto de investigación "El feedback correctivo escrito directo e indirecto en la adquisición y aprendizaje del español como lengua extranjera", inscrito en el Fondo Nacional de Desarrollo Científico y Tecnológico (FONDECYT) 1140651 (2014-2017).

\section{RESUMEN}

El objetivo de este artículo es identificar el léxico disponible de un grupo de aprendices de español como lengua extranjera (ELE) de nivel de competencia lingüística $\mathrm{B} 1$ en tres centros de interés: la ciudad; alimentos y bebidas, y medios de transporte, mediante la aplicación de una encuesta de disponibilidad léxica. Los resultados indican que el centro de interés que impulsó mayor cantidad de entradas léxicas fue alimentos y bebidas. Se puede inferir que el léxico de los aprendices de ELE en función de los centros de interés en estudio se encuentra acorde con el nivel de proficiencia B1 - según las nociones básicas del Marco Común Europeo de Referencia para las lenguas (MCER) - y que avanza hacia una consolidación en los posteriores niveles. La aplicabilidad que tiene este tipo de investigación se ve reflejada en el ámbito de la enseñanza de segundas lenguas, específicamente, en la selección adecuada de vocabulario para las clases de ELE por parte de los docentes, quienes deben considerar las unidades léxicas tanto disponibles como frecuentes.

Palabras clave: léxico disponible, disponibilidad léxica, centros de interés, competencia léxica, español como lengua extranjera, enseñanza de lenguas extranjeras

\section{Abstract}

The aim of this research article is to determine the lexical competence of a group of learners of Spanish as a Foreign Language (SFL) at B1 proficiency level in three interest centers: foods and beverages, the city, and means of transportation. The study was conducted by using a Lexical Availability poll. The results show that the interest center that triggered the biggest amount of lexical entries was foods and beverages. It can be inferred that the lexical competence of learners of SFL corresponds to a B1 level of proficiency - according to the Common European Framework of Reference for languages (CEFR) - and that it moves towards consolidation in upper levels. How applicable this kind of study may be belongs to the field of second language teaching, specifically an adequate selection of vocabulary to use in SFL lessons by teachers - as they must consider both available and frequent lexical items.

Keywords: available lexicon, lexical availability, interest centers, lexical competence, Spanish as a foreign language, foreign language teaching

Received: 2016-09-29 / Accepted: 2017-10-25

DOI: $10.17533 /$ udea.ikala.v23n03a07 


\section{RÉSUMÉ}

Le but de cet article est d'identifier le lexique disponible d'un groupe d'apprenants de l'espagnol comme langue étrangère (ELE) du niveau de compétence linguistique B1 dans trois centres d'intérêt -la ville, la nourriture et les boissons, et les moyens de transport-, par l'application d'une enquête de disponibilité lexicale. Les résultats indiquent que le centre d'intérêt qui a déclenché le plus grand nombre d'entrées lexicales était la nourriture et les boissons. On peut en déduire que le lexique des stagiaires ESL selon les centres d'intérêt étudiés est conforme au niveau de compétence B1 selon les notions de base du MCER pour les langues et évoluant vers la consolidation dans les niveaux ultérieurs. L'applicabilité de ce type de recherche est reflétée dans le domaine de l'enseignement des langues secondes, en particulier dans la sélection adéquate du vocabulaire des classes d'ELE par les enseignants, qui devraient considérer à la fois des unités lexicales disponibles et fréquentes.

Mots-clés: lexique disponible, disponibilité lexicale, centres d'intérêt, compétence lexicale, espagnol langue étrangère, enseignement des langues étrangères 


\section{Introducción}

En el ámbito de la adquisición de segundas lenguas (ASL), los primeros estudios sobre léxico disponible comenzaron en Francia en los años cincuenta del siglo Xx, con la recopilación de palabras que aparecían con frecuencia en textos escritos, con la finalidad de elaborar un manual de enseñanza de la lengua francesa como segunda lengua, dirigido a grupos de inmigrantes. López Morales (1993) atribuye este trabajo al francés René Michéa (1953). Décadas más tarde y con procedimientos más avanzados y sistemáticos, Carcedo (1998), profesor de la Universidad de Turkuen, Finlandia, analizó la disponibilidad léxica en alumnos de español como lengua extranjera (ELE).

El interés por la cultura hispana y el español como segunda lengua ha aumentado tanto en Europa como en otras partes del mundo. Esto puede constatarse en que el número de alumnos que estudia ELE crece cada año (Instituto Cervantes, 2016). En este contexto, es relevante realizar estudios sobre el léxico disponible en los aprendices ELE, porque el léxico que se enseña y que se aprende debe ser el reflejo de la lengua en uso, una lengua inserta en un plano comunicativo y real.

El aprendizaje del léxico debe ser activo por parte de los aprendientes de ELE. Debe integrar múltiples procesos cognitivos, con énfasis en la negociación de significado en situaciones reales o auténticas. Desde esta perspectiva, en el contexto de enseñanza y aprendizaje del léxico de ELE, surgen preguntas como: ¿qué léxico enseñar?, ¿qué centros de interés son los que cobran mayor relevancia?

A partir de lo anterior, la identificación de léxico disponible mediante la aplicación de encuestas de disponibilidad léxica se configura como un instrumento que evalúa el léxico.

En relación con los planteamientos anteriores, se formuló como pregunta de investigación: ¿cuál es el léxico disponible que presentan los aprendices de ELE de nivel B1 cuando son evaluados mediante una encuesta de disponibilidad léxica en función de tres centros de interés: la ciudad; alimentos y bebidas, y medios de transporte?

\section{Objetivo}

Esta investigación tiene como objetivo identificar el léxico disponible de un grupo de aprendices de ELE de nivel de competencia lingüística B1, acerca de tres centros de interés: la ciudad; alimentos y bebidas, y medios de transporte, mediante la aplicación de una encuesta de disponibilidad léxica.

\section{Fundamentos teóricos}

\section{Léxico y competencia léxica}

El dominio del léxico por los aprendientes de segundas lenguas es un componente fundamental en el proceso de adquisición para el desarrollo de la competencia léxica, mediante distintas habilidades comunicativas (hablar, leer, escribir y escuchar). La interacción entre los hablantes constituye un medio para el intercambio y la negociación de significados. Sin léxico, ningún hablante puede comunicarse eficazmente con otro en un escenario comunicativo.

Una definición general sobre competencia léxica es la propuesta por Lahuerta y Pujol (1996), quienes la definen como "la capacidad para relacionar formas con significados y utilizarlas adecuadamente" (p. 121). Para el Instituto Cervantes (2002), esta competencia es el conocimiento del vocabulario de una lengua y la capacidad para utilizarlo.

En esta misma línea se destaca la teoría de la competencia léxica, postulada por Marconi (2000), quien la identifica, de manera general, con la capacidad de usar las palabras. De esta forma, el conocimiento del léxico se configura como un vehículo de significado para una comunicación efectiva y exitosa entre los hablantes de segundas lenguas. 
Hernández Muñoz (2005), apoyándose en los fundamentos postulados por Marconi (2000), afirma que la apropiación de las palabras y su uso implica, a su vez, dos tipos de competencias:

\begin{abstract}
1) inferencial: conocimiento de la red de conexiones que se establece entre una palabra y el resto de las voces y las expresiones lingüísticas del mismo sistema. Implica destrezas como la inferencia semántica, la paráfrasis, la definición y el hallazgo de sinónimos. 2) referencial: capacidades para proyectar las palabras en el mundo real, principalmente: nombrando (reconocer un objeto y encontrar la palabra) y aplicando (entender una palabra dada y escoger el objeto) (p. 40).
\end{abstract}

Esta teoría de la competencia léxica también incluye la existencia de dos tipos de léxico: por una parte, el léxico semántico (reconocimiento del objeto a la palabra $)^{1}$ y, por otra, el léxico de educto (representaciones fonéticas y gráficas de una palabra). El léxico disponible se encuentra vinculado al primer tipo de competencia léxica (la inferencial), 508 ya que en esta se enumeran palabras relacionadas semánticamente.

\section{Léxico disponible}

Uno de los primeros investigadores en referirse al léxico disponible fue Michéa (1953), quien postuló que:

El vocablo disponible se caracteriza porque se presenta en la mente del hablante de forma inmediata y natural cuando se trata un determinado tema. Es una palabra que, sin ser necesariamente frecuente, vive potencialmente en el hablante y se actualiza en cuanto se producen ciertas asociaciones (p. 340).

En otros términos, cada vez que aprendientes de segunda lengua están frente a una situación de comunicación y deben utilizar sus habilidades comunicativas, todas las entradas léxicas que acuden a su memoria en ese momento corresponden al léxico disponible, el cual está constituido por aquellas

1 Asignación arbitraria de un nombre a una realidad física o abstracta. palabras que un hablante puede activar inmediatamente en su memoria, según las necesidades derivadas de la producción lingüística (Ferreira, Salcedo y Del Valle, 2014). López Morales (1983) definió este tipo de vocabulario como aquel conjunto de unidades léxicas de contenido semántico y concreto. El mismo autor (1999), a la luz de sus investigaciones, también se refiere al lugar donde reside el léxico disponible y afirma que

Existe en el lexicón mental una serie de términos que no se actualizan a menos que sean necesarios para comunicar una información muy específica. Se trata de un léxico disponible, cuyo estudio no puede emprenderse manejando frecuencias, porque este factor es pertinente sólo en el caso de las actualizaciones léxicas efectivas, no de las potenciales (p. 11).

Este tipo de léxico es parcelado, no es un todo que esté disponible, sino que lo están solo ciertas entradas agrupadas en categorías independientemente de su frecuencia.

En cuanto al lexicón mental, se puede definir también como "el almacén de palabras (representaciones mentales) que contiene la información semántica o conceptual, la información sintáctica y las unidades léxicas" (Aitchison, 2003). Luque (1998) ahonda en este último término, refiriéndose al lexicón como una realidad mental paralela a la realidad física que conocemos como "mundo", y distingue en el lexicón dos componentes: unidades y redes. En suma, el léxico es almacenado en la mente de cada hablante, espacio en el cual se establecen relaciones de significados que permiten desarrollar la competencia léxica. Otra definición general corresponde a la propuesta por Garnham (1992), quien concibe el lexicón mental como "estado mental del conocimiento acerca de las palabras que incluye cómo se deletrea una palabra, cómo se pronuncia, cómo se inserta en el discurso, qué significa, etc." Así, abarca distintos niveles de la lengua, desde el fonológico hasta el pragmático.

Desde una perspectiva neurolingüística, Bartol (2010) contribuye con una explicación relevante para el estudio del léxico disponible: 
El léxico disponible supone un nuevo enfoque sobre el léxico. No se trata de las palabras utilizadas al producir determinados textos; sino de las palabras que, organizadas, están en nuestro lexicón mental y están "disponibles" para ser utilizadas cuando las circunstancias comunicativas lo requieran; cuando un estímulo active el nodo cerebral adecuado (p. 91).

Siguiendo este postulado, cobra gran relevancia el rol del contexto comunicativo, el cual configura las circunstancias para utilizar el léxico que se halla disponible en el lexicón mental de los aprendientes de segundas lenguas.

\section{Disponibilidad léxica}

Constituye un instrumento que permite determinar y conocer cuál es el léxico disponible de los aprendientes. Saralegui y Tabernero (2008) afirman que la disponibilidad léxica es un enfoque de investigación, dentro de la lingüística, que tiene como objetivo la recogida y el posterior análisis del léxico disponible de una determinada comunidad de habla.

Fernández-Merino (2012) relaciona la disponibilidad y la competencia léxica con los programas de inmersión lingüística, dado que, desde una perspectiva de enseñanza, proporcionan datos útiles para la selección y la nivelación del léxico en las clases de español como segunda lengua.

A partir de la recogida de los datos, se pueden realizar comparaciones a nivel léxico con otras comunidades de hablantes y disponer de una base estadística que facilite la organización de la enseñanza de las unidades léxicas (Samper Hernández, 2002).

En esta misma línea, para Jiménez Berrío (2012), en la disponibilidad léxica, el foco de atención se encuentra centrado en el aprendizaje integrado de contenidos, por medio de la enseñanza de vocabulario. Por lo tanto, priman, para la adquisición del léxico, programas de enseñanza basados en la aplicación de encuestas que identifiquen las unidades más y menos disponibles.
En el contexto de las evaluaciones de disponibilidad léxica, los centros de interés son temas que agrupan un conjunto de entradas léxicas. Samper, Bellón y Samper-Hernández (2003) sostienen que son "unos estímulos temáticos relativamente amplios que persiguen provocar las respuestas de los sujetos entrevistados" (p. 47). Por ejemplo, los muebles de la casa, objetos colocados sobre la mesa, la educación, la política, la salud, etc. Para Sánchez Saus (2011), son ideas fuerza que funcionan como palabras clave y cuyo objetivo es mostrar las palabras que tienen algún vínculo con un tema determinado.

Los centros de interés también pueden ser considerados como hiperónimos, que permiten evidenciar el léxico disponible, residente en el lexicón mental, sobre un tema en particular de carácter concreto o abstracto.

\section{Metodología}

\section{Diseño del estudio}

Esta investigación de disponibilidad léxica es un estudio mixto, con un diseño no experimental de tipo descriptivo transversal. El objetivo de este tipo de diseño es observar, identificar y describir el comportamiento de los aprendientes, sin ningún tipo de intervención, es decir, identificar el léxico disponible de un grupo de aprendientes de segundas lenguas en un momento determinado.

\section{Participantes}

En este estudio participaron veintiún aprendices de español como segunda lengua, que llevaban alrededor de cuatro meses en Chile. Pertenecían al "Programa de Español como Lengua Extranjera", impartido por la Universidad de Concepción, ${ }^{2}$ en

2 El programa "promueve la enseñanza, investigación y difusión del Español como Lengua Extranjera en sus variantes latinoamericanas. Con el propósito de mejorar la comunicación en Español como LE, el programa integra la enseñanza de tareas interculturales con aprendizajes colaborativos en ambientes tecnoló- 
el contexto de intercambio estudiantil. Asistían a clases del programa tres horas a la semana.

Los estudiantes participantes contaban con cierta competencia lingüística del español, ya que habían estudiado formalmente el idioma o incursionado en él de manera autónoma, pero sistemática, por lo que se hallaban en el nivel de proficiencia B1, según los niveles de competencia lingüística (Instituto Cervantes, 2002).

Los estudiantes cursaban las siguientes carreras: Ingeniería Mecánica, Ingeniería Comercial, Ingeniería Industrial, Ingeniería de Materiales, Arquitectura, Hidrología, Ingeniería Civil, Ingeniería Ambiental, Lenguas Modernas, y Literatura y Traducción.

Sus edades oscilaban entre los 20 y 23 años.

Del total de los participantes, la muestra estuvo constituida por 11 sujetos del género femenino, equivalente al 52,3\% del total de la muestra, y 10 sujetos de género masculino, correspondiente al $46,7 \%$ restante.

\section{Los centros de interés}

Los centros de interés fueron seleccionados de acuerdo con el plan curricular definido por el Marco Común Europeo de Referencia para las lenguas (MCER). Estos centros presentan mayor idoneidad con el nivel de proficiencia B1, según la revisión de las nociones básicas.

La ciudad constituye una noción relevante para los aprendientes de ELE, quienes deben desplazarse por ella a menudo, conocer sus calles y elementos que la conforman, con la finalidad de resolver situaciones de la vida diaria, como salir de compras, realizar un trámite, reunirse con un amigo, etc.

gicos sociales y culturales [sic]". Para mayor información véase Universidad de Concepción (s. a.).
Los alimentos y bebidas son un tema de primera necesidad para los extranjeros, dado que deben identificarlos para adquirirlos para su consumo, por ejemplo, en supermercados, restaurantes, mercados, entre otros.

Los medios de transporte se enmarcan como centros de interés altamente demandados por los estudiantes de segundas lenguas. Conocer qué medios de transporte hay en su nueva ciudad y de cuáles pueden disponer es elemental para su vida académica y personal.

\section{Encuesta de disponibilidad léxica}

En la primera parte de la encuesta (véase anexo), cada aprendiente proporcionó datos personales y académicos. En la segunda parte, los aprendices completaron el léxico sobre los tres centros de interés: la ciudad; alimentos y bebidas, y medios de transporte.

Los alumnos dispusieron de dos minutos por cada tema.

Trabajaron con listas abiertas, presentadas como un juego lúdico cronometrado, es decir, escribieron unidades que en ese momento se activaron en su memoria respecto a los temas solicitados.

\section{Procesamiento de los datos}

Una vez recopiladas las entradas léxicas, fueron procesadas en el programa Dispogen, el cual realiza un análisis mixto de los datos: cuantitativo (promedio de respuestas por centro de interés, número de palabras diferentes - NPD-e índice de cohesión - IC - el cual indica el grado de coincidencia u homogeneidad entre las respuestas de los aprendientes), y cualitativo (índice de disponibilidad léxica - IDL — o grado de latencia de un ítem en léxico en la memoria de los aprendientes, frecuencia absoluta y frecuencia relativa de las entradas léxicas). 
Los ítems léxicos se ingresaron en formato de texto (.txt), en número singular y con letra minúscula.

\section{Análisis de los resultados}

Los resultados incluyen, en primer lugar, el análisis del promedio de respuestas por centro de interés; en segundo lugar, el NPD; en tercer lugar, el IC, y en cuarto y último lugar, el IDL por cada centro de interés.

\section{Promedio de respuestas por centro de interés}

La figura 1 presenta el promedio de respuestas por cada centro de interés con respecto a la encuesta de disponibilidad léxica. El eje temático que registró el promedio mayor correspondió a alimentos $y$ bebidas, con un promedio de 26,4 respuestas. Una razón por la que los aprendientes del nivel de proficiencia B1 expresaron mayor cantidad de entradas léxicas en este eje temático podría deberse a que los alimentos y bebidas se enmarcan como un tema de primera necesidad para los hablantes extranjeros cuando llegan a otro país. Todo este léxico identificado reflejaría, en este centro, hábitos de consumo básico o gustos individuales de los aprendices de ELE.

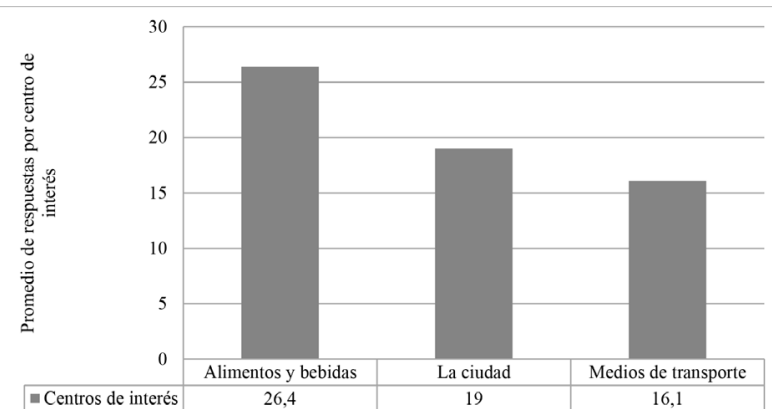

Figura 1 Promedio de respuestas por centro de interés Fuente: Elaboración propia.

El centro que registró el menor promedio de respuestas fue medios de transporte, equivalente a un 16,1. Esto se debería a que posiblementela cantidad de ítems léxicos relativos a los medios de trans- porte disponibles sería más limitada, a diferencia de los tipos de alimentos y bebidas, y de los elementos de la ciudad, en los cuales existiría mayor cantidad de entradas léxicas.

El porcentaje restante correspondió al centro de interés la ciudad, con un promedio de 19 respuestas. Este centro generó varias entradas léxicas. Esto se puede atribuir a que, como el aprendiz de este nivel es un usuario independiente, es capaz de comprender ideas y almacenar léxico relacionado con áreas de experiencias que le son relevantes, tales como elementos que conforman su nueva ciudad, lugares de compras, lugares de interés, entre otros.

\section{Número de palabras diferentes}

La figura 2 evidencia que el centro de interés alimentos y bebidas registró el mayor NPD (98, equivalente a un $40 \%$ ), seguido del eje temático la ciudad (92, correspondiente a un $37 \%$ ). Finalmente, el centro de interés que registró la menor cantidad de palabras diferentes correspondió a medios de transporte (57, o sea el $23 \%$ ). Los aprendices no manifestaron gran variación en las entradas léxicas correspondientes a este último centro. Una razón podría deberse a que no existe un alto número de entradas disponibles asociadas a la locomoción.

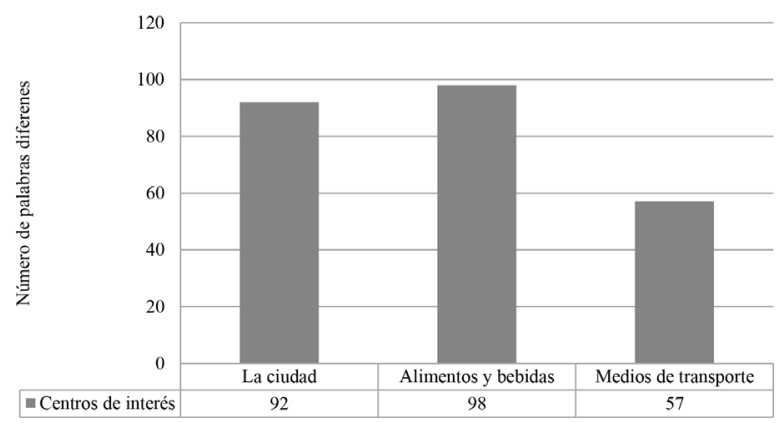

Figura 2 Número de palabras diferentes por centro de interés

Fuente: Elaboración propia. 


\section{Índice de cohesión}

La figura 3 indica los centros temáticos que registraron mayor grado de coincidencia entre las respuestas de los participantes en la encuesta de disponibilidad léxica. El centro de interés medios de transporte registró un IC de 0,15 . Pese a que en este tema los aprendientes no impulsaron gran cantidad de léxico, hubo coincidencia. Al parecer, los hablantes de nivel $\mathrm{B} 1$ en este centro de interés compartirían una base léxica similar, almacenada en su lexicón mental.

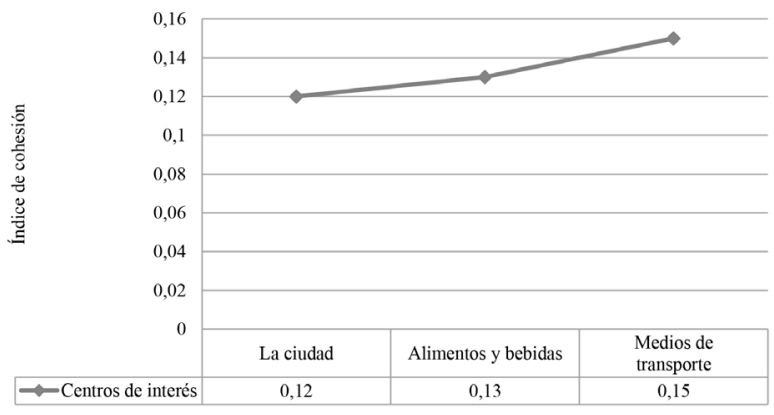

Figura 3 Índice de cohesión por centro de interés Fuente: Elaboración propia.

El centro de interés que registró un menor IC correspondió a la ciudad, con 0,12; en otras palabras, los aprendientes de este tema coincidieron en varias entradas léxicas, al igual que en el centro de interés alimentos y bebidas, el cual registró un IC de 0,13.

Se puede afirmar que en los centros de interés no existe una diferencia abismal en el grado de coincidencia de las entradas léxicas respecto a los centros de interés. Esto podría deberse a que los participantes almacenarían en su memoria una base léxica en común, acorde con el nivel de proficiencia B1.

\section{Índice de disponibilidad léxica}

\section{Disponibilidad léxica en centro de interés la ciudad}

Las entradas léxicas disponibles por los participantes en este eje temático ascendieron a 399.
La tabla 1 presenta el IDL, la frecuencia, ${ }^{3}$ el porcentaje de aparición y la frecuencia acumulada de las diez entradas léxicas más registradas.

Las entradas léxicas que resultaron más disponibles fueron calle, edificio y gente. El léxico calle presentó una frecuencia de 18 , es decir, se repitió en 18 de las 21 encuestas, y alcanzó la máxima disponibilidad de los participantes, un 0,8344; o sea, dentro del lexicón mental de los aprendientes existió una alta probabilidad de utilizar este vocablo. El resto de las entradas léxicas manifestaron menor índice de disponibilidad. Así lo demostraron los siguientes ítems: plaza, auto y supermercado. Todos estos tuvieron una disponibilidad inferior al 0,2785.

El ítem léxico gente apareció como una entrada bastante disponible dentro de las encuestas. Llama la atención este resultado, ya que en este centro de interés los aprendices tienden a activar, en su memoria, léxico alusivo a lugares y elementos propios de una ciudad, posiblemente relacionado con la nueva vida llevada como extranjeros.

\section{Disponibilidad léxica en centro de interés alimentos y bebidas}

El total de entradas léxicas registradas en este centro de interés correspondió a 556. Este eje destacó respecto a los otros, ya que se situó como el que más activó entradas léxicas en la memoria de los hablantes de ELE. En la tabla 2 se presenta el IDL, la frecuencia, el porcentaje de aparición y la frecuencia acumulada de las diez entradas léxicas más disponibles de este eje temático.

En el centro de interés alimentos y bebidas, las entradas léxicas que resultaron más disponibles en el lexicón mental de los participantes fueron agua $(0,4342)$, manzana $(0,3769)$ y vaso $(0,3542)$.

3 La frecuencia se refiere al número de veces que apareció un ítem léxico en todas las encuestas, a diferencia de la frecuencia acumulada, que explicita la suma o total acumulado de todas las frecuencias hasta la entrada léxica que se está observando. 
Tabla 1 IDL, frecuencia, porcentaje de aparición y frecuencia acumulada en centro de interés la ciudad

\begin{tabular}{l|c|c|c|c}
\hline Vocablo & IDL* & Frecuencia & Porcentaje de aparición (\%) & Frecuencia acumulada \\
\hline Calle & 0,8344 & 18 & 85,71 & 0,0451 \\
Edificio & 0,5027 & 13 & 61,90 & 0,0776 \\
Gente & 0,4008 & 13 & 61,90 & 0,1102 \\
Casa & 0,3950 & 11 & 52,38 & 0,1378 \\
Tienda & 0,3921 & 14 & 66,66 & 0,1729 \\
Semáforo & 0,3480 & 12 & 57,14 & 0,2030 \\
Parque & 0,3371 & 13 & 61,90 & 0,2355 \\
Plaza & 0,2785 & 9 & 42,85 & 0,2581 \\
Auto & 0,2373 & 8 & 38,09 & 0,2781 \\
Supermercado & 0,2268 & 9 & 42,851 & 0,3007 \\
\hline
\end{tabular}

*IDL: Índice de disponibilidad léxica.

Tabla 2 IDL, frecuencia, porcentaje de aparición y frecuencia acumulada en centro de interés alimentos y bebidas

\begin{tabular}{l|c|c|c|c}
\hline Vocablo & IDL* $^{*}$ & Frecuencia & Porcentaje de aparición (\%) & Frecuencia acumulada \\
\hline Agua & 0,4342 & 15 & 71,42 & 0,0269 \\
Manzana & 0,3769 & 13 & 61,90 & 0,0503 \\
Vaso & 0,3542 & 11 & 52,38 & 0,0701 \\
Jugo & 0,3541 & 11 & 52,38 & 0,0899 \\
Cerveza & 0,3238 & 10 & 47,61 & 0,1079 \\
Pisco** & 0,3048 & 8 & 38,09 & 0,1223 \\
Carne & 0,3034 & 15 & 71,42 & 0,1492 \\
Pan & 0,2976 & 10 & 47,61 & 0,1672 \\
Fruta & 0,2926 & 10 & 47,61 & 0,1852 \\
Verdura & 0,2841 & 10 & 47,61 & 0,2032 \\
\hline
\end{tabular}

${ }^{*}$ IDL: Índice de disponibilidad léxica.

** La Real Academia Española (RAE) (2018) define "pisco" como: aguardiente de uva.

Llama la atención la alta disponibilidad del ítem vaso en este centro de interés, dado que es más probable que hubiese aparecido, según los estudios realizados en los centros de interés del "Proyecto Panhispánico”, en objetos colocados en la mesa

4 Se conoce como el "Proyecto Panhispánico" al proyecto dirigido por Humberto López Morales (1973), que tiene como objetivo estudiar el léxico disponible de los hablantes nativos del español, a ambos lados del Atlántico, que todavía no hayan iniciado sus estudios para la comida o, en su defecto, en la cocina y sus utensilios; sin embargo, apareció con una alta disponibilidad, que lo lleva a ocupar el tercer lugar en el eje temático alimentos y bebidas. Al parecer, los aprendices de ELE con un nivel de competencia B1 calificarían este tipo de objetos como elementales a la hora del consumo alimenticio.

universitarios. Según la página web DispoLex 30, su finalidad principal es elaborar diccionarios de disponibilidad léxica de distintas zonas hispanohablantes. 
Con respecto a las entradas léxicas fruta y verdura, las cuales destacan como menos disponibles, constituyeron léxico hiperónimo, ya que muchos vocablos se circunscribieron a estas, como ocurrió con manzana.

\section{Disponibilidad léxica en centro de interés medios de transporte}

En la tabla 3 se presenta el IDL, la frecuencia, el porcentaje de aparición y la frecuencia acumulada de las entradas léxicas en este centro, que resultó el menos productivo de los tres en estudio. El total de entradas registradas en las encuestas fue 337.

En el centro de interés medios de transporte resultaron más disponibles los ítems léxicos bus, avión, y micro. Entre los menos disponibles de la tabla 3, destacaron barco, colectivo y taxi.

Los aprendientes impulsaron entradas léxicas alusivas a todos los tipos de medios de transporte: transporte aéreo, avión; transporte terrestre, bus, tren, micro, entre otros; transporte marítimo, barco.

Llama la atención que si bien la entrada léxica avión fue altamente frecuente, la primera entrada léxica disponible en el lexicón mental de los hablantes es bus. Una razón podría ser que este medio de transporte terrestre sería uno de los más accesibles, cercanos y disponibles para los aprendientes.

\section{Discusión}

En este artículo se han presentado los principales planteamientos teóricos, la metodología de investigación y los resultados del estudio sobre léxico que se llevó a cabo con un grupo de aprendices de ELE en la Universidad de Concepción. El objetivo principal de esta investigación fue identificar el léxico disponible que tenían los aprendices de ELE con un nivel de proficiencia $B 1$, respecto a tres centros de interés: la ciudad; alimentos y

Tabla 3 IDL, frecuencia, porcentaje de aparición y frecuencia acumulada en centro de interés medios de transporte

\begin{tabular}{l|c|c|c|c}
\hline Vocablo & IDL $^{*}$ & Frecuencia & Porcentaje de aparición (\%) & Frecuencia acumulada \\
\hline Bus & 0,6662 & 17 & 80,95 & 0,0504 \\
Avión & 0,6427 & 21 & 100 & 0,1127 \\
Micro $^{a}$ & 0,6371 & 17 & 80,95 & 0,1632 \\
Tren $^{a}$ & 0,5826 & 19 & 90,47 & 0,2195 \\
Auto $^{b}$ & 0,5586 & 14 & 66,66 & 0,2611 \\
Bicicleta $^{\text {Coche }}{ }^{\mathrm{a}}$ & 0,4699 & 15 & 71,42 & 0,3056 \\
Barco $^{\text {Colectivo }}{ }^{\mathrm{d}}$ & 0,3379 & 11 & 52,38 & 0,3382 \\
Taxi & 0,2833 & 13 & 61,90 & 0,3768 \\
\hline
\end{tabular}

*IDL: Índice de disponibilidad léxica.

a. "Medio de transporte terrestre. Sinónimo de bus" (Definición ABC, 2018).

b. Automóvil: "Dicho principalmente de los vehículos que pueden ser guiados para marchar por una vía ordinaria sin necesidad de carriles y llevan un motor, generalmente de combustión interna o eléctrico, que los propulsa". (RAE, 2018). "En el español de Chile se utiliza el término auto como apócope".

c. Para la RAE (2018), "coche se refiere a automóvil destinado al transporte de personas y con capacidad no superior a siete plazas. Coche es un término que no se usa en el español de Chile para referirse a automóvil. Tiene mayor uso en otras partes de Latinoamérica".

d. "Transporte público (generalmente automóvil) de pasajeros con recorrido fijo" (Reverso Diccionario, 2018). 
bebidas, y medios de transporte, mediante una encuesta de disponibilidad léxica.

En cuanto al promedio de respuestas por centro de interés, la categoría que arrojó mayor cantidad de léxico resultó ser alimentos y bebidas, mientras que la menos productiva fue medios de transporte; sin embargo, esta última resultó ser la que mostró mayor grado de coincidencia entre las respuestas de los aprendientes de ELE. Estos hallazgos son coincidentes con la investigación realizada por Magnúsdóttir (2012), quien estudió la disponibilidad léxica en alumnos islandeses de ELE. En este contexto, primero apareció el centro alimentos y bebidas, seguido de la ciudad y, finalmente, medios de transporte.

En relación con el IDL, los hallazgos de esta investigación también coinciden con los de Magnúsdóttir (2012), en que la unidad léxica más disponible de alimentos y bebidas corresponde a agua. En cuanto a la ciudad, su estudio determinó como unidad léxica altamente disponible coche, en contraste con nuestros resultados, que determinaron que calle era el léxico con mayor grado de latencia.

En medios de transporte nuevamente existe disonancia. En Magnúsdóttir (2012) aparece que el léxico más disponible fue coche, mientras que nuestros hallazgos arrojaron que era bus. Frente a este escenario se puede señalar la incidencia de la variable geográfica, dado que en el español de Chile no se utiliza el término "coche". En aprendices eslovenos, el promedio de palabras en los tres centros de interés apareció en el mismo orden (Sifrar, 2012).

Estos resultados son similares, pero no iguales a los encontrados por López Rivero (2008). Sus hallazgos apuntan a que el centro de interés que agrupó un mayor léxico disponible por aprendientes de ELE de nivel B1 correspondió a la ciudad, argumentando que posiblemente los informantes se encontraban inmersos en la vida urbana, en el contexto del intercambio estudiantil, por lo cual generaron mayor léxico en relación con alimentos y bebidas.

\section{Conclusiones}

Esta investigación ha dejado en evidencia, por medio de la aplicación de la encuesta de disponibilidad léxica, el léxico disponible, residente en el lexicón mental, que tienen los aprendientes de ELE pertenecientes a un nivel B1, sobre los tres centros de interés en estudio.

La disponibilidad léxica resultó ser un índice relevante, ya que permitió identificar los ítems léxicos más disponibles de los hablantes y con ello contribuir con una descripción aproximativa de la competencia léxica que poseen los aprendientes de ELE en el nivel B1.

En este sentido, es necesario contar con mecanismos que permitan conocer las palabras disponibles que requieren las personas en contextos determinados, pues el conocimiento del léxico es una competencia lingüística básica para el proceso de aprendizaje y para el proceso comunicativo de una segunda lengua (Lin, 2012).

Es preciso destacar que las entradas léxicas con mayor índice de disponibilidad no resultaron siempre con la máxima frecuencia. Esto, debido a que la frecuencia indica el número de repeticiones y la disponibilidad da cuenta de cuán latente es una entrada léxica en el lexicón mental de los aprendientes.

A la luz de lo anterior, se puede concluir, en relación con estos tres centros de interés, que el léxico disponible por este grupo de aprendices de ELE se encuentra acorde con el nivel B1 de competencia lingüística, según la revisión de las nociones específicas del MCER (Instituto Cervantes, 2002) y que avanza hacia una clara consolidación en los posteriores niveles de competencia lingüística. Además, que la disponibilidad léxica es un indicador útil que permite seleccionar léxico tanto para el tratamiento de los contenidos y materiales, como para la evolución de la competencia léxica. 


\section{Referencias}

Aitchison, J. (2003). Words in The Mind: An Introduction to The Mental Lexicon (3. ${ }^{\mathrm{a}}$ ed.). Oxford and Cambridge: Blackwell.

Bartol, J. A. (2010). Disponibilidad léxica y selección del vocabulario. En R. M. ${ }^{a}$ Castañer Martín y V. Lagüéns Gracia (Eds.), De moneda nunca usada. Estudios

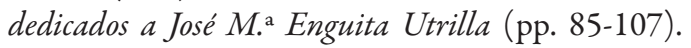
Zaragoza: Institución Fernando el Católico.

Carcedo, A. (1998). Sobre las pruebas de disponibilidad léxica para estudiantes de español LE. RILCE, 14(2), 205-224.

Definición ABC. (2018). Micro. Recuperado de https:// www.definicionabc.com/general/micro.php

Dispolex. (2018). Recuperado de http://www.dispolex.com/

Fernández-Merino Gutiérrez, P. (2012). Presencia del léxico disponible de inmigrantes en glosarios específicos de vocabulario. Cuadernos Comillas, (2), 1-18. Recuperado de http://www.fundacioncomillas.es/cuader nos-comillas/cuadernos/lexico/pdf/cuaderno.pdf

Ferreira, A., Salcedo, P. y Del Valle, M. (2014). Estudio de disponibilidad lexica en el ámbito de las matemáticas. Revista Estudios Filológicos,(54), 69-84.

Garnham, A. (1992). Psycholinguistics: Central topics. Londres, Nueva York: Routledge.

Hernández Muñoz, N. (2005). La disponibilidad léxica: una herramienta fronteriza para el estudio del léxico en Lingüística y Psicología. En Estudios sobre adquisición del lenguaje. (pp. 942-954): Salamanca. Ediciones Universidad de Salamanca.

Instituto Cervantes, (2002). Common European Framework of Reference for Languages: Learning, Teaching, Assesment. Madrid, España: Artes Gráficas Fernández Ciudad, S. L.

Instituto Cervantes (2016). El español, una lengua viva. Informe 2012. Recuperado de http://www.cervantes. es/imagenes/File/prensa/El\%20espaol\%20una\%20 lengua\%20viva.pdf

Jiménez Berrío, F. (2012). Aprendizaje integrado de contenidos y español para inmigrantes desde la perspectiva de la disponibilidad léxica: enseñanza del vocabulario. En: R. Breeze, F. Jiménez Berrío, C. Llamas Saíz, C. Martínez Pasamar y C. Tabernero Sala (Eds.), Teaching approaches to CLIL / Propuestas docentes en AICLE (pp. 149-163). Pamplona: Servicio de Publicaciones de la Universidad de Navarra.
Lahuerta J. y Pujol, M. (1996). El lexicón mental y la enseñanza del vocabulario. La enseñanza del léxico español como lengua extranjera. Madrid: Editorial Iberoamericana Carlos Segoviano.

Lin, J. (2012). El estudio de disponibilidad léxica de los estudiantes chinos de español como lengua extranjera. Marco ELE Revista de Didáctica Español como Lengua Extranjera, (14), 1-14. Recuperado de http:// marcoele.com/descargas/14/lin-disponibilidad_ lexica.pdf

López Morales, H. (1983). Lingüística estadística. En H. López, Introducción a la lingüistica actual (pp. 209225). Madrid: Playor.

López Morales, H. (1993). Los estudios de disponibilidad léxica: pasado y presente. Boletín de Filología de la Universidad de Chile, XXXV, 245-259.

López Morales, H. (1999). Léxico disponible de Puerto Rico. Madrid: Arco Libros.

López Rivero, E. (2008). Estudio de disponibilidad léxica en 43 estudiantes de ELE. Memoria del Máster en Enseñanza del Español como Lengua Extranjera. España: Universidad Antonio de Nebrija.

Luque, J. de D. (1998). Introducción a la tipología léxica. En B. Gallardo (ed.), Temas de lingüistica y gramática, (pp. 122-145). Valencia: Universidad de Valencia.

Magnúsdóttir, S. (2012). Disponibilidad léxica en alumnos de español como lengua extranjera. Estudio sobre el léxico disponible en alumnos de ELE en la secundaria en Islandia (Tesis de Maestría en Instrucción en Español, Universidad de Islandia).

Marconi, D. (2000). La competencia léxica. Madrid: Antonio Machado Libros.

Michéa, R. (1953). Mots fréquents et mots disponibles. Un aspect nouveau de la statistique du langage. Les Langues modernes, (47), 338-344.

Real Academia Española (RAE). (2018). Diccionario de la lengua española (23. ${ }^{\mathrm{a}}$ ed.). Consultado en http:// www.rae.es/

Reverso Diccionario. (2018). Colectivo. Recuperado de http://diccionario.reverso.net/espanol-definiciones/ colectivo

Samper, J. A., Bellón, J. J., y Samper Hernández, M. (2003). El proyecto de estudio de la disponibilidad léxica en español. En R. Ávila et al (Eds.), Pautas y pistas en el análisis del léxico hispano (americano) (pp. 27-139). Vervuert: Iberoamericana. 
Samper Hernández, M. (2002). Disponibilidad léxica en alumnos de español como lengua extranjera. Málaga: ASELE.

Sánchez Saus, M. (2011). Bases semánticas para el estudio de los centros de interés del léxico disponible. Disponibilidad léxica de informantes extranjeros en las universidades andaluzas (Tesis, Universidad de Cádiz (UCA), Departamento de Filología).

Saralegui, C., y Tabernero, C. (2008). Aportación al proyecto panhispánico de léxico disponible: Navarra. En I. Moreno, M. Casado Velarde y R. González Ruiz (Eds.), Actas del XXXVII Simposio Internacional de la Sociedad Española de Lingüistica (SEL) (pp. 745762). Pamplona, España: Servicio de Publicaciones de la Universidad de Navarra.

Sifrar, M. (2012). Análisis comparativo de la disponibilidad léxica en español como lengua extranjera (ELE) y lengua materna (ELM). Marco ELE Revista de Didáctica Español como Lengua Extranjera, (15), 1-19. Recuperado de http://marcoele.com/descargas/15/ sifrar-disponibilidad_lexica.pdf

Universidad de Concepción (s. a.). "Programa de Español como Lengua Extranjera. Presentación". Recuperado de http://ele.udec.cl/

\section{Anexo. Encuesta de disponibilidad léxica}

Instrucciones: Complete los siguientes datos que se le solicitan.

\section{Datos del informante:}

1. Sexo: Hombre Mujer

2. Edad:

3. País de origen:

4. Lengua materna:

5. Universidad de origen:

6. Carrera:

7. Tiempo que lleva viviendo en Chile:

Instrucciones: A continuación, complete las siguientes listas con todas las palabras del español que se activen en su memoria. Usted dispone de un total de seis minutos, dos minutos por cada tema. Respete el tiempo cuando se le indique que cambie de lista. No olvide escribir con letra clara.

\begin{tabular}{l|l|l}
\hline \multicolumn{1}{c|}{ La ciudad } & \multicolumn{1}{c|}{ Alimentos $\mathbf{y}$ bebidas } & \multicolumn{1}{c}{ Medios de transporte } \\
\hline 1. & 1. & 1. \\
\hline 2. & 2. & 2. \\
\hline 3. & 3. & 3. \\
\hline$[\ldots]$ & & \\
\hline 35. & 35. & 35. \\
\hline
\end{tabular}

How to reference this article: Blanco San Martín, L. P., y Ferreira Cabrera, A. A. (2018). Léxico disponible en tres centros de interés de aprendices de español como lengua extranjera. Íkala, Revista de Lenguaje y Cultura, 23(3), 505-517. DOI: 10.17533/udea.ikala.v23n03a07 